\title{
Limitaciones en el desarrollo de la actividad matemática en la escuela básica: el caso de la aritmética escolar*
}

\author{
Limitations in the development of the mathematical activity \\ in primary school: the case of the school arithmetic

\section{Limitaçoes no desenvolvimento da atividade matemática} \\ na escola primária: o caso de uma aritmética escolar
}

\author{
Lorena Espinoza1, Joaquim Barbé ${ }^{2}$, Grecia Gálvez \\ ${ }^{1}$ Universidad de Santiago de Chile. \\ ${ }^{2}$ Grupo Félix Klein, Universidad de Santiago de Chile.
}

\begin{abstract}
RESUMEN
La investigación que presentamos estudió el problema de los escasos niveles de desarrollo de la actividad matemática en estudiantes chilenos de educación básica. El artículo se centra en el caso de la aritmética escolar. Basándonos en la Teoría Antropológica de lo Didáctico (Chevallard, 1999) y en la Teoría de Situaciones Didácticas (Brousseau, 1994), analizamos los contenidos y aprendizajes matemáticos nucleares de los programas oficiales en el eje de aritmética. Continuamos con la observación y análisis de procesos de enseñanzaaprendizaje en unidades temáticas de aritmética, en escuelas de la Región Metropolitana, y finalizamos con el análisis de condiciones y restricciones institucionales impuestas por las escuelas al desarrollo de la actividad matemática. El trabajo realizado, que aborda las dimensiones curricular, fáctica e institucional, nos permite postular lo que podría ser considerado un fenómeno didáctico por su grado de generalidad y su vinculación directa con una estrategia específica de enseñanza de las matemáticas (Brousseau, 1983). El fenómeno consiste en que en muchos casos la escuela inhibe, de forma no intencionada y muy a su pesar, las potencialidades matemáticas que tienen los niños.
\end{abstract}

Palabras clave: educación básica, aprendizaje matemático, aritmética escolar.

\begin{abstract}
This research analyzes the problem of the low levels of the development of the Mathematics Activity, reached by Chilean students at the end of the primary school. The article focuses on the case of School Arithmetic. Using the Anthropological Theory of Didactics (Chevallard, 1999) and the Theory of Didactic Situations (Brousseau, 1994), the investigation began with the analysis of nuclear mathematical contents and learning of the current official primary school programs in the area of arithmetic. Next, we observed and analyzed the teaching-learning processes developed in thematic units of arithmetic in primary schools of the Metropolitan Region. We finished with the analysis of the institutional conditions and restrictions on the mathematic activities imposed by the schools. This paper approaches the curricular, factual and institutional dimensions. It allows us to postulate what might be considered to be a didactic phenomenon by its degree of generality and its direct entailment with a specific strategy of education of mathematics (Brousseau, 1983). The phenomenon is that, in many cases, in spite of its good intentions, the school inhibits the mathematical potentialities that the children have.
\end{abstract}

Key words: primary school, mathematical learning, school arithmetic.

* Trabajo inserto dentro del Proyecto Regular de Investigación FONDECYT No 1050234, financiado por CONICYT. 


\section{RESUMO}

A investigação que apresentamos estudou o problema dos escassos níveis de desenvolvimento da atividade matemática em estudantes chilenos de Educação Primaria. O artigo centra-se no caso da aritmética escolar. Baseandonos na Teoria Antropológica do Didático (Chevallard, 1999), e na Teoria de Situações Didáticas (Brousseau, 1994), analisamos os conteúdos e aprendizagens matemáticas nucleares dos programas oficiais no eixo de aritmética. Continuamos com a observação e análise de processos de ensino-aprendizagem em unidades temáticas de aritmética, em escolas da Região Metropolitana, e finalizamos com a análise de condições e restrições institucionais impostas pelas escolas ao desenvolvimento da atividade matemática. $\mathrm{O}$ trabalho realizado, que aborda as dimensões curricular, fática e institucional, nos permite postular o que poderia ser considerado um fenômeno didático por seu grau de generalidade e seu vínculo direto com uma estratégia específica de ensino das matemáticas (Brousseau, 1983). O fenômeno consiste em que, em muitos casos, a escola inibe, de forma não intencionada e muito a sua pesar, as potencialidades matemáticas que têm as crianças.

Palavras chave: educação primaria, aprendizagem matemática, aritmética escolar.

\section{INTRODUCCION}

Desde comienzos de la década de los 90 distintas iniciativas han sido desarrolladas en el ámbito educativo nacional, con el propósito de potenciar el sistema de enseñanza y lograr un mejoramiento sustantivo del aprendizaje de los estudiantes. Un hito fundamental lo ha constituido la Reforma Educativa que, además de incidir sobre las condiciones laborales de los docentes y sobre las condiciones de aprendizaje de los alumnos, ha generado importantes cambios en el currículo en todos los sectores de aprendizaje (Cox, 2003).

La implementación de la Reforma Educativa ha incluido un conjunto de acciones orientadas a apoyar la tarea docente, impulsadas desde el Ministerio de Educación, como el desarrollo de redes de actualización de docentes de diferentes niveles y disciplinas, la distribución de diversos materiales curriculares, la organización de pasantías y sistemas de intercambio con instituciones educativas del país y del extranjero, el desarrollo de Programas de Postítulo para profesores de Segundo Ciclo Básico que otorgan la mención en distintos subsectores del currículo, etc.

Pese a los esfuerzos realizados hasta la fecha, no se han logrado consolidar los cambios en las formas de enseñar y de aprender que han sido propuestos. Los últimos resultados de las pruebas nacionales de medición de la calidad de la educación, SIMCE, muestran que el progreso académico de los estudiantes en el subsector de matemáticas no ha sido generalizado (Informe Nacional de Resultados SIMCE 2007, sobre los resultados de los alumnos que cursaron $4^{\circ}$ y $8^{\circ}$ básico en el año 2007). En términos generales, los niveles de aprendizaje logrados por la mayoría de los alumnos son todavía muy bajos, tanto en comparación con el currículum como en relación a los estándares internacionales (TIMSS-MINEDUC, 2004; PISA-MINEDUC, 2004).

Informaciones sobre ítems liberados por el Ministerio muestran que hay conocimientos matemáticos elementales que los alumnos de $8^{\circ}$ básico no manejan. Por ejemplo, el ítem de la prueba SIMCE de $8^{\circ}$ : “¿Cuál es el área de una región rectangular si su largo es $60 \mathrm{~cm}$ y su ancho un tercio de la medida anterior?" fue respondido correctamente sólo por el 30\% de los estudiantes. En esa misma prueba, el ítem "Un kilo de asado cuesta \$ 2.400. Si compro $3 / 4 \mathrm{~kg}$ de asado, ¿cuánto pago?” fue respondido correctamente sólo por el $45 \%$ de los estudiantes.

Resta pendiente la tarea de avanzar hacia una implementación efectiva del currículo que garantice el logro de aprendizajes de calidad. Esta tarea no es simple, ya que para alcanzarla es necesario superar varias dificultades del sistema educativo, en sus distintos 
niveles: micro, meso y macro ${ }^{1}$. Señalaremos dos que, a nuestro juicio, son centrales. La primera está relacionada con la complejidad y exigencias que plantea el nuevo currículo a los docentes y alumnos de básica de nuestro país, equiparable en muchos casos a las de países de la OCDE (Barbé y Espinoza, 2004). La segunda consiste en lo laborioso que resulta para los docentes modificar sus prácticas pedagógicas habituales. En la actualidad, debido en gran medida a su escasa formación matemática y didáctica, los profesores tienen serias limitaciones para ir más allá de una enseñanza de la aritmética centrada en la utilización mecánica de algoritmos. Para que profesores y profesoras logren transformar efectivamente sus prácticas pedagógicas se requiere fortalecer su capacidad docente a través de un trabajo de desarrollo profesional en el que actualicen y profundicen sus conocimientos, especialmente en el ámbito de las didácticas específicas (OCDE, 2004).

En el contexto de la gestión de la enseñanza básica, la política ministerial vigente desde los 90 se ha focalizado fuertemente en dar apoyo al primer ciclo ( $1^{\circ}$ a $4^{\circ}$ años). El Programa de las 900 Escuelas estuvo dirigido a este ciclo durante los primeros ocho años de su operación, centrándose en lenguaje y matemáticas. El propio Ministerio reconoce que, desde la década de los 90, ha habido un escaso desarrollo de acciones dirigidas al segundo ciclo, tanto en lenguaje como en matemáticas.

Considerando los antecedentes señalados, no es de extrañar que aparezca cierto grado de desarticulación entre ambos ciclos. Más allá de existir quiebres o rupturas, como las encontradas en el Proyecto Fondecyt $N^{\circ} 1020342$ entre la enseñanza básica y media (Espinoza y Barbé, 2004), se presume que existe un débil incremento de los niveles de aprendizaje alcanzados por los alumnos en los distintos cursos del $2^{\circ}$ ciclo de educación

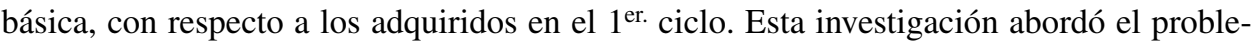
ma del escaso nivel de aprendizaje matemático que logran los estudiantes de enseñanza

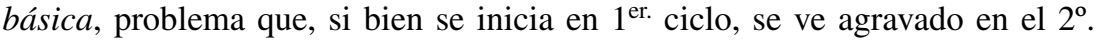

Desde la perspectiva teórica que asumimos, este problema puede formularse en términos de la actividad matemática ${ }^{2}$ que alumnos y profesores realizan en básica. Es probable que, en ambos ciclos, las tareas matemáticas que el profesor propone realizar sean aisladas y rutinarias, no incorporen suficientemente la resolución de problemas, no requieran de decisiones por parte del alumno en cuanto al conocimiento útil para resolverlas -ya que éste viene dado en el enunciado de la tarea-, no demanden que el alumno explique y controle lo que hace, tampoco que contraste distintos procedimientos o técnicas para evaluar la pertinencia y limitaciones de las mismas. En $2^{\circ}$ ciclo se incorporan progresivamente conocimientos matemáticos más complejos pero, considerando el escaso incremento de los niveles de aprendizaje durante el $2^{\circ}$ ciclo que hemos señalado, podríamos suponer que el tipo de actividad matemática que los alumnos realizan es similar al que ejecutan en el primer ciclo. Los resultados de nuestra investigación prece-

1 El nivel micro corresponde a la escuela y, más específicamente, a la sala de clases; el nivel meso, al entorno más cercano a la escuela: comuna, departamento provincial de educación, padres, familias; y el nivel macro, al Ministerio de Educación, universidades, la sociedad en su conjunto.

2 Entendemos por actividad matemática una actividad de estudio de campos de problemas, que pueden provenir de la propia matemática, de otras disciplinas o del entorno social y cultural. En torno a estos problemas se va construyendo un conjunto de técnicas para abordarlos y resolverlos y, asociados a éstas, los elementos tecnológicos y teóricos que permiten describirlas, explicarlas, contrastarlas y, en último término, justificarlas (Chevallard, 1999). 
dente sobre la actividad matemática que se desarrolla en $8^{\circ}$ básico apoyan esta hipótesis (Espinoza y Barbé, 2004).

Esta investigación tuvo como propósito identificar y caracterizar factores de la educación básica chilena, en el ámbito de la educación matemática, que obstaculizan el progreso en el estudio de las matemáticas en el segundo ciclo y que dificultan que los estudiantes alcancen los niveles de logro de aprendizaje que los actuales programas proponen.

Para llevarla a cabo fue necesario caracterizar el estudio de la matemática en el $1^{\text {er. }} \mathrm{y}$ $2^{\circ}$ ciclo de educación básica y luego realizar los respectivos contrastes. El marco teórico utilizado se constituye a partir de nociones fundamentales del Enfoque Epistemológico de Didáctica de las Matemáticas (Brousseau, 1986), con especial énfasis en Teoría Antropológica de lo Didáctico de Yves Chevallard (1999) y en Teoría de Situaciones de Guy Brousseau (1990).

La metodología de investigación se enmarca dentro de la corriente de la antropología cognitiva (Sensevy, 1999). Se trata de una investigación cualitativa, basada en el estudio clínico de los sistemas didácticos (Leutenegger, 2000), y que utiliza técnicas etnográficas.

Postulamos la existencia de factores de naturaleza curricular, referidos a los conocimientos matemáticos que según el marco curricular y los programas se debe estudiar en estos ciclos; de naturaleza fáctica, en cuanto a las prácticas docentes y a las exigencias hechas a los alumnos; y de orden institucional, en cuanto a las formas de organización general de la enseñanza en cada ciclo.

Por cuestiones de espacio, en este artículo presentamos los resultados de la investigación referidos al ámbito de la aritmética escolar, dejando para otra ocasión los relacionados con geometría y tratamiento de la información.

\section{MARCO TEORICO DE LA INVESTIGACION}

Nuestra investigación se enmarca en la corriente del Enfoque Epistemológico en Didáctica de las Matemáticas, iniciado por Guy Brousseau en la década de los 70. Este enfoque se construye a partir de la Teoría de Situaciones de Guy Brousseau, que actúa como núcleo generador del paradigma, y de los aportes de Yves Chevallard (Transposición didáctica; Teoría Antropológica de lo Didáctico), Michèle Artigue (Reproductibilidad de Situaciones), Régine Douady (Teoría Herramienta-Objeto) y otros autores. Este enfoque constituye un marco teórico coherente, legitimado, que dispone de resultados sólidamente probados y es seguido por una parte importante de la comunidad científica internacional que investiga en esta área.

Nos apoyamos especialmente en los aportes de Teoría de Situaciones de Guy Brousseau (1990) y de Teoría Antropológica de lo Didáctico, TAD, de Yves Chevallard (Chevallard, 1999; Espinoza et al., 2005). Estas herramientas teóricas nos permitieron estudiar el problema del escaso nivel de aprendizaje matemático que logran los estudiantes de educación básica.

El enfoque epistemológico considera la didáctica de las matemáticas como la "ciencia de las condiciones de creación y difusión de los conocimientos matemáticos útiles a los hombres y a sus instituciones" (Brousseau, 1994). Postula que la investigación de cualquier 
problemática didáctica debe incorporar el análisis de los conocimientos matemáticos tal cual son reconstruidos en las instituciones de enseñanza, y su correspondiente proceso de transposición didáctica (Chevallard, 1985). Este proceso consiste en las sucesivas adaptaciones que deben experimentar los conocimientos matemáticos para ser enseñados. Se sostiene que aprender matemáticas consiste esencialmente en hacer matemáticas y, por tanto, en la realización de una práctica. Aprender matemáticas comporta la realización de un proceso que sitúa en el centro del quehacer del que aprende el estudio de problemas articulados entre sí. Según la Teoría Antropológica de lo Didáctico este proceso de estudio está constituido por distintas dimensiones o momentos del trabajo que realizan profesor y alumnos, que van desde la exploración auténtica de problemas, a la justificación y sistematización de lo matemáticamente construido, pasando por el trabajo de rutinización de los procedimientos que permite a los estudiantes no solo resolverlos, sino que plantear nuevos problemas.

Un criterio central para elaborar situaciones de aprendizaje consiste en elegir aquellas que constituyan situaciones fundamentales de aprendizaje, en el sentido de G. Brousseau (1990). Esto es, una situación problemática que el niño enfrenta inicialmente sin la intervención directa del profesor, a través de su interacción con un medio que le devuelve información sobre la adecuación de sus acciones frente al problema, cada vez que lo manipula. En este medio intervienen ciertas condiciones o variables didácticas que, al ser controladas adecuadamente por el profesor, "obligan" al niño a progresar en sus acciones hasta lograr la construcción del conocimiento matemático esperado.

La Teoría de Situaciones Didácticas distingue los momentos en los que el alumno trabaja sin la intervención directa del profesor de los momentos en que el profesor interviene directamente sobre el trabajo de los niños. En el primer caso, los alumnos se enfrentan a la exploración y estudio de problemas mediante la interacción con sus compañeros y con un medio. Este tipo de situaciones, denominadas adidácticas, resultan ser esenciales para que los estudiantes construyan conocimiento matemático significativo. En el segundo caso, se requiere de la intervención del profesor para institucionalizar, es decir, sistematizar y legitimar el conocimiento, reconociéndolo como matemático. En este tipo de situaciones la participación del alumno también es central, pero se requiere la gestión directa del profesor, y por ello se denominan situaciones didácticas. Parte fundamental de la acción del alumno la constituye la validación de la producción de soluciones a las situaciones problemáticas abordadas. Por validación se entiende el proceso a través del cual un alumno puede determinar si su producción ha sido adecuada o no y argumentar el por qué de su adecuación.

La Teoría Antropológica de lo Didáctico, por su parte, adopta un punto de vista institucional de la problemática didáctica, situándola dentro del marco más general de las prácticas humanas. Para modelizar el conocimiento matemático utiliza la noción de organización o praxeología matemática. Una organización matemática, en adelante OM, está compuesta por cuatro categorías de elementos: tipos de problemas, técnicas matemáticas, elementos tecnológicos y elementos teóricos. Esta modelización hace referencia a una constitución estática o estructural de la actividad matemática.

Los procesos de enseñanza y aprendizaje de las matemáticas aparecen como medios para la construcción de OM's en la escuela; ambos procesos están incluidos dentro de un proyecto común que esta teoría denomina el proceso de estudio de las matemáticas (Chevallard et al., 1997). Este proceso es modelizado a través de la Teoría de los Momentos 
Didácticos, modelización que hace referencia al aspecto dinámico o funcional de la actividad matemática, que puede ser descrita a través de seis momentos. Los momentos se distribuyen de forma dispersa a lo largo del proceso de estudio, pueden aparecer más de una vez en dicho proceso e incluso pueden coexistir entre sí. Ellos son: momento del primer encuentro, exploratorio, del trabajo de la técnica, tecnológico-teórico, de la institucionalización y de la evaluación.

A pesar de la relación intrincada que existe entre lo matemático y lo didáctico, parece razonable que el análisis de la actividad matemática comience por el análisis de las OM's que emergen de esta actividad (Chevallard, 2004). En nuestra investigación partimos analizando las organizaciones matemáticas, tales como han sido propuestas en el marco curricular y los actuales programas de estudio. Este análisis curricular aborda, además, el grado de coherencia y completitud de las OM's y la articulación de éstas dentro del programa de enseñanza establecido.

Cada $\mathrm{OM}$ es el resultado de un proceso de estudio realizado en el seno de una institución determinada. Dicha institución impone restricciones específicas para la realización del estudio. Por ello, es necesario complementar el análisis curricular con el de las organizaciones didácticas que son construidas por profesor y alumnos al realizar el estudio de las OM's propuestas. Este segundo tipo de análisis, que aborda la dimensión fáctica, es realizado mediante las nociones de praxeología didáctica del profesor y del alumno, la teoría de los momentos didácticos y el contrato didáctico.

La noción de praxeología didáctica del profesor permite describir la actividad docente en términos de su componente práctica (el "saber-hacer" o praxis) y su componente teórica y justificadora (el "saber" o logos). Podemos distinguir dos niveles de análisis de dicha práctica. El primero se centra en estudiar los tipos de tareas y técnicas didácticas que utiliza el profesor para organizar y dirigir el proceso de estudio de una OM específica en una institución de enseñanza determinada. Al lado de las técnicas didácticas, se sitúan, en un segundo nivel, las tecnologías y elementos teóricos que permiten al profesor describir, justificar, organizar y dirigir su práctica. Análogamente, la noción de praxeología didáctica del alumno nos permite describir su actividad en términos de su componente práctica y su componente teórica.

El estudio de una OM forma parte de un proyecto común entre profesor y alumnos, establecido dentro del marco más amplio de una institución de enseñanza específica. En el desarrollo y evolución de dicho proyecto se van estableciendo, a través de un proceso de negociación más o menos implícito entre los participantes, las normas que van a regir el funcionamiento y desarrollo del estudio. Se van negociando y estableciendo las cláusulas del contrato didáctico, que adjudican y distribuyen las responsabilidades tanto del profesor como de los alumnos (Brousseau, 1990). Estas reglas delimitan qué cosas se pueden hacer durante el estudio de una OM determinada y cuáles no están permitidas. Así, cuando existe un proceder más o menos generalizado entre los estudiantes, es probable que dicho comportamiento obedezca a ciertas normas del contrato didáctico, o a transgresiones de éstas, las que han sido designadas por Brousseau como rupturas de contrato.

El contrato didáctico está influenciado por restricciones que la institución de enseñanza le impone. La práctica del profesor se encuentra sometida a las imposiciones institucionales que gobiernan los aspectos generales que afectan el entorno del estudio. Estas imposiciones ejercen fuertes influencias sobre la práctica del alumno. Por tanto, 
es necesario completar los anteriores análisis con un tipo de estudio que constituye el tercer nivel de análisis de nuestra investigación y que aborda la dimensión que hemos designado como institucional.

\section{METODOLOGIA}

Dado que el propósito de nuestra investigación era caracterizar factores inherentes al sistema educativo que inciden sobre el desarrollo de la actividad matemática en la educación básica, se requería de una comprensión amplia y profunda de dicho Sistema. Esto nos condujo a realizar un trabajo de corte cualitativo, basado en el estudio clínico de los sistemas didácticos (Leutenegger, F, 2000), que utiliza técnicas etnográficas.

El problema es abordado desde las tres dimensiones ya señaladas: curricular, fáctica e institucional. Para el análisis curricular se estudiaron los programas de $1^{\circ}$ y $2^{\circ}$ ciclo básico, los libros de texto oficiales y material informativo sobre las últimas pruebas SIMCE de $4^{\circ}$ y $8^{\circ}$ básico y sobre la prueba TIMSS de $8^{\circ}$ básico (2002). El material curricular fue segmentado en unidades temáticas, las que, previa clasificación dentro de los ejes temáticos del $1^{\circ}$ y $2^{\circ}$ ciclo básico, fueron analizadas en términos de organizaciones matemáticas. En cada una de las OM's obtenidas fueron identificados cuatro tipos de elementos: tipos de tareas, técnicas, tecnología y teoría. El grado de coherencia de cada OM se determinó analizando en qué medida los elementos tecnológicos y teóricos explican y justifican sus tareas y técnicas. El grado de completitud de cada OM se determinó en función de la presencia o ausencia de los cuatro tipos de elementos que la componen, y el grado de articulación entre OM's se determinó estableciendo los elementos que comparten, y la relación entre ellos. En base a las inconsistencias detectadas, tanto en el análisis curricular como en los estudios encontrados en la revisión bibliográfica, se anticipó qué conflictos podrían aparecer en la dimensión fáctica.

Para el análisis de la dimensión fáctica fueron observados y analizados procesos didácticos relativos a la enseñanza y el aprendizaje matemático en $4^{\circ}$ y $5^{\circ}$ básico. Dicho análisis nos permitió contrastar las formulaciones derivadas del análisis curricular. El material analizado se obtuvo mediante observaciones de campo, entrevistas y cuestionarios. La codificación de dicho material se realizó a partir de categorías generales preestablecidas, provenientes de la TAD. Una vez codificado, se analizó usando técnicas etnográficas. Este análisis se complementó con el realizado para $8^{\circ}$ básico en el Proyecto Fondecyt $\mathrm{N}^{\mathrm{o}}$ 1020342. Se escogieron establecimientos municipalizados y particulares subvencionados. En cada uno de los cursos $\left(4^{\circ}\right.$ y $\left.5^{\circ}\right)$ fueron seleccionadas tres unidades temáticas de los programas de estudio, para ser observadas de acuerdo a criterios emanados del análisis curricular. En total, se observaron 12 procesos, cada uno de alrededor de un mes de clases. Las observaciones realizadas fueron respaldadas por grabaciones en video. Como información complementaria, se consignó el libro de texto utilizado, y se recogió cuadernos de algunos alumnos, apuntes del profesor, los instrumentos de evaluación de la unidad y las hojas de respuesta de algunos alumnos, la prueba diagnóstico y guías de trabajo dadas por el profesor.

En base a los videos, las notas tomadas por el observador y el material complementario recogido, se codificó el contenido matemático estudiado y la forma en que se realizó el proceso de estudio de cada unidad. Según el rol que desempeñaba dentro del proceso 
cada una de las unidades de análisis observadas, ésta se clasificó en una de las cuatro categorías de la OM, y en uno de los seis momentos didácticos. De esta manera se logró una descripción detallada del proceso de estudio que daba cuenta tanto del contenido matemático tratado como de la forma en que se construyó dicho contenido.

Sobre la base de la descripción del proceso en cada unidad temática y las dimensiones que describen el saber del profesor, se construyó el protocolo de dos entrevistas al profesor, una que se aplicó antes de iniciar el proceso de enseñanza y la otra al terminarlo. Una vez aplicadas las entrevistas, fueron identificados cuatro tipos de elementos: tipos de tareas didácticas, técnicas didácticas, tecnología didáctica y teoría didáctica. La información obtenida en las entrevistas fue contrastada y complementada con la descripción de la práctica del profesor a partir de los registros.

Además, se elaboró un cuestionario que se aplicó a los alumnos, considerando lo que debieran ser capaces de hacer una vez terminado el proceso. A partir de la descripción del proceso para cada unidad temática, el análisis de la entrevista al profesor y el cuestionario a los alumnos, se infirió una descripción del contrato didáctico, identificando patrones de interacción.

Para el análisis de la dimensión institucional, a partir de las descripciones de los contratos didácticos fueron detectadas normas o patrones que parecían corresponder a restricciones institucionales. Se realizaron entrevistas a directivos y focus group con los profesores de básica de cada establecimiento, y se recogió documentación de carácter reglamentario. El análisis de resultados de estudios cualitativos sobre el sistema educativo chileno sirvió para triangular la información obtenida y describir los contratos institucionales en el primer y segundo ciclo básico.

Comparando los resultados del análisis de la dimensión curricular, fáctica e institucional en función de las coincidencias y diferencias en las OM's, los momentos didácticos, la praxeología del profesor, la praxeología del alumno, el contrato didáctico y el contrato institucional, se realizó una caracterización del estudio de la matemática en cada uno de los ciclos considerados. La comparación entre ambas caracterizaciones permitió, posteriormente, contrastar y verificar las hipótesis del estudio.

\section{PRINCIPALES RESULTADOS}

A continuación presentamos los principales resultados obtenidos en el ámbito específico de la aritmética, considerando el estudio de los números, de las operaciones y de las propiedades de éstas.

\subsection{CONTENIDOS Y APRENDIZAJES MATEMATICOS NUCLEARES DEL EJE DE ARITMETICA DEL CURRICULO}

La primera fase de la investigación consistió en realizar un análisis del Marco Curricular y de los Programas de Estudio, en relación a los aprendizajes matemáticos nucleares de cada nivel. Se caracterizó la estructura y dinámica de las principales organizaciones matemáticas (OM) que se propone enseñar en estos cursos, así como las restricciones que impone cada una de ellas a la actividad matemática que es posible llevar a cabo en las escuelas básicas. Este estudio, de carácter curricular, supuso además 
analizar los libros de texto más difundidos en el sistema, los sistemas de evaluación oficiales (SIMCE) y material curricular de apoyo para el docente.

\section{En relación al primer ciclo básico}

Los actuales programas oficiales de matemática en el $1^{\text {er }}$ ciclo básico distinguen cuatro ejes temáticos que organizan los contenidos que se deben enseñar, en una lógica progresiva de construcción. Estos son: números, operaciones, formas y espacio y resolución de problemas. Este último eje aparece como transversal a los tres restantes.

En cuanto a las OM's que, según la postura oficial se deben enseñar en este ciclo, se detecta una propuesta rica, y actualizada en términos didácticos y matemáticos. Se trata de una propuesta coherente y articulada de temas matemáticos que van siendo construidos en los cuatro ejes temáticos definidos. En los programas se entregan elementos precisos para orientar la enseñanza de contenidos matemáticos específicos, abundantes ejemplos y actividades de aprendizaje genéricas para proponer a los niños. Sin embargo, el programa elude la presentación de definiciones precisas y utiliza un lenguaje familiar para los niños, al parecer para facilitar la comprensión de las nociones, sacrificando cierta rigurosidad matemática. Aparecen así ambigüedades entre, por ejemplo, número, dígito, cifra y cantidad. La terminología usada puede llevar a confusiones posteriores puesto que sugiere interpretaciones equívocas, como la de "recta numerada" (Programa $1^{\circ}$ Básico, pág. 147). Del mismo modo, por momentos se confunde lo que es un concepto con su representación. Las técnicas matemáticas sugeridas se apoyan fuertemente en la intuición y conocimientos culturales de los niños, especialmente en el caso de las fracciones. Las ideas claves de estos programas son: (a) Construir los números naturales vinculados fuertemente al sentido de la cantidad que representan, por lo que en un primer momento los números surgen a propósito de la tarea de contar y cuantificar colecciones, y posteriormente se inicia la tarea de su representación a través del estudio del sistema de numeración decimal; (b) Construir las operaciones aritméticas de manera integrada y con sentido, en vez de considerarlas de forma aislada, para luego buscar la ocasión de articularlas. Así, se construye la adición apoyándose en la sustracción y viceversa, constituyendo ambas operaciones el campo de problemas aditivos. De igual forma, la división aparece como la operación que se resuelve apoyándose en la multiplicación, conformando ambas operaciones el campo de problemas multiplicativos; (c) Construir progresivamente los algoritmos convencionales de cálculo, apoyándose en la comprensión de procedimientos que explicitan las propiedades del sistema de numeración decimal, al mismo tiempo que permiten profundizar en las propiedades de las operaciones; (d) Construir las fracciones como números que permiten resolver problemas de reparto equitativo en los que la cantidad a repartir es de naturaleza fraccionable y no es múltiplo de la cantidad de participantes del reparto. Asimismo, las fracciones aparecen como números que permiten resolver problemas de cuantificación de partes de la unidad, cuando ésta ha sido fraccionada equitativamente; (e) En geometría, estudiar las relaciones entre figuras y no sólo las propiedades al interior de cada tipo de figura; (f) Recurrir a nociones y procedimientos geométricos para resolver problemas relativos al espacio; (g) Estudiar problemas, más que sólo resolverlos, como centro de la actividad matemática que desarrollan los niños.

Sin embargo, estas importantes y valiosas proposiciones no han sido suficientemente incorporadas en los libros de texto, y casi nada en las prácticas docentes habituales. 
Las pruebas SIMCE de los últimos años se han ido alineando cada vez más con esta propuesta curricular.

\section{En relación al segundo ciclo básico}

El análisis curricular detectó que en los programas de estudio del $2^{\circ}$ ciclo básico varía tanto la forma de organización como las categorías de estructuración utilizadas en los programas de $1^{\text {er }}$ ciclo. Así, se pierde la estructura organizada por ejes de los programas de $1^{\text {er }}$ ciclo; los aprendizajes esperados no aparecen explícitos en un solo lugar del documento, sino que están entremezclados con los contenidos mínimos, las orientaciones didácticas y las actividades sugeridas. Tampoco contienen indicadores de aprendizaje; hay una escasa explicitación del progreso en el estudio matemático al interior de cada curso y entre cursos, y las "actividades genéricas" de $1^{\mathrm{er}}$ ciclo, pasan a tener el carácter de "actividades sugeridas", en $2^{\circ}$ ciclo.

A lo largo de este ciclo van apareciendo cuatro OM en torno a números; éstas son: naturales, fracciones positivas, decimales positivos y números con signo (que incluye los enteros). La OM en torno a los naturales incorpora el estudio de la familia de grandes números, que responde al problema del tratamiento de información cuantitativa expresada con dichos números. La idea es hacer visible el orden de magnitud de una cantidad a partir de referentes más cercanos y significativos. Se enfatiza mucho menos que en $1^{\text {er }}$ ciclo el carácter decimal de nuestro sistema de numeración. De hecho, la estructura y las leyes de formación del sistema de numeración decimal (SND) dejan de ser un objeto de estudio en sí mismos, para dar curso a un trabajo de utilización de los números para comunicar, registrar y producir información cuantitativa. En este sentido, el tratamiento de magnitudes y la búsqueda de expresiones equivalentes usando distintas unidades de medida se convierten en una problemática de mayor relevancia que en $1^{\text {er }}$ ciclo. Las técnicas matemáticas sugeridas se apoyan menos en la intuición y conocimientos culturales de los niños, mientras que van apareciendo elementos tecnológicos del ámbito de las matemáticas, sobre todo en el caso de las fracciones.

La estrategia didáctica propuesta para el estudio de las fracciones positivas se basa en que las fracciones son "signos" que permiten dar cuenta de acciones de fraccionamiento, con un estatuto de número: se puede operar con ellas y ordenarlas. Ya no se habla de números mixtos sino de fracciones propias e impropias. Aparecen todas las fracciones, a la vez que se introducen nuevos significados de ellas: como razón y operador. Aparece también la tarea de comparar fracciones con distintos denominadores, que requerirá de la amplificación y simplificación para ser resuelta.

$\mathrm{La} \mathrm{OM}$ en torno a los decimales positivos se empieza a estudiar en $6^{\circ}$ básico y continúa en $7^{\circ}$. Estos números aparecen en los programas a propósito del problema de la exactitud en la medición. Sin embargo, el estudio propuesto no retoma suficientemente la estructura utilizada en $1^{\text {er }}$ ciclo para estudiar el SND. El mayor problema que existe en los programas de $2^{\circ}$ ciclo, se presenta en $8^{\circ}$ básico. Aparecen aquí, abrupta y tardíamente, los llamados por el programa "números con signo". Al parecer, se trata del estudio de los números racionales, aunque se da gran énfasis a los números enteros. Esta estrategia tiene como consecuencia una falta de consolidación del trabajo en este eje, que no permite su integración adecuada dentro de la OM más global de números. 
En el ámbito del estudio de las operaciones y sus propiedades, aparecen seis OM insuficientemente articuladas entre sí: adición y sustracción con fracciones, y problemas asociados; multiplicación y división con fracciones, y problemas asociados; adición y sustracción con decimales, y problemas asociados; multiplicación y división con decimales, y problemas asociados; adición y sustracción con números con signo, y problemas asociados; multiplicación y división con números con signo, y problemas asociados. En todas estas OM's se propone una construcción articulada de cada operación con su inversa, pero las actividades sugeridas siguen la lógica de aislar ambas operaciones. Se pierde así el trabajo propuesto en el $1^{\circ}$ ciclo en esta dirección, en particular toda la terminología usada para delimitar y caracterizar los distintos tipos de problemas como aditivos y multiplicativos.

En términos generales, podemos sostener que los programas del $1^{\mathrm{er}}$ ciclo básico son más específicos, estructurados y sistemáticos que los del $2^{\circ}$ ciclo. En $2^{\circ}$ ciclo se observan unas OM's más ricas que las propuestas en $1^{\mathrm{er}}$ ciclo, ya que la componente tecnológico-teórica de éstas incorpora elementos nuevos en relación a las OM's de $1^{\text {er }}$ ciclo. Sin embargo, en su mayoría estos elementos están muy poco desarrollados y aparecen implícitos en las actividades, por lo que se prevé que los profesores deberán completarlos, pese a las escasas herramientas con que cuentan para hacerlo. Especial mención merecen en este sentido las OM's propuestas en el ámbito de la geometría.

No obstante estas grandes diferencias, las OM's de ambos ciclos comparten una característica común, esencial a la hora de su correspondiente enseñanza y aprendizaje. Tanto unas como otras tienen una componente práctica, esto es, tipos de tareas asociadas a los aprendizajes esperados y técnicas o procedimientos para resolverlas, que no generan la necesidad de recurrir a un conocimiento matemático nuevo. Dicha necesidad permitiría que los niños se encontraran con el "sentido" o "razón de ser" de los conocimientos, y no sólo con su aplicación. Los problemas planteados tampoco provocan el cuestionamiento sobre cuándo es útil y pertinente utilizar ciertas técnicas y no otras, ni la necesidad de explicar por qué dichas técnicas funcionan frente a un determinado problema y por qué fracasan frente a otro. Por lo general, las actividades propuestas permiten ilustrar los conocimientos en distintos y variados contextos, pero no posibilitan su construcción real por parte del estudiante. Ambos tipos de OM's siguen propiciando la forma tradicional de enseñanza de las matemáticas y, aunque aparecen problemas de ilustración novedosos, la actividad de los niños sigue centrada en la aplicación rutinaria de procedimientos previamente enseñados o "mostrados" por el profesor.

\subsection{ANALISIS DE PROCESOS DE ENSEÑANZA Y APRENDIZAJE DESARROLLADOS EN TORNO A LA ARITMETICA EN ESCUELAS BASICAS DE LA REGION METROPOLITANA}

Para realizar el análisis de la dimensión fáctica de la investigación, esto es, la forma en que profesores y niños realizan el estudio de las matemáticas en la escuela básica, fueron observados 12 procesos de estudio, 6 en el ámbito de la aritmética y 6 en el de la geometría. El diseño experimental contempló dos establecimientos municipalizados y dos particulares subvencionados, con el fin de considerar la influencia de restricciones institucionales correspondientes a estos dos tipos de dependencia administrativa. En cada uno de los cursos $\left(4^{\circ}\right.$ y $\left.5^{\circ}\right)$ se escogieron tres unidades temáticas presentes en los programas de estudio y asociadas a los tres ejes temáticos (números, operaciones y geometría), para ser observadas de acuerdo a criterios emanados del análisis curricular. 

forma:

La observación de los procesos en cada establecimiento se realizó de la siguiente

\begin{tabular}{|c|c|c|c|}
\hline \multicolumn{2}{|c|}{ Est. Municipal 1 } & \multicolumn{2}{c|}{ Est. Part. Subvencionado 1 } \\
\hline Est. Municipal 2 & \multicolumn{2}{c|}{ Est. Part. Subvencionado 2 } \\
\hline $4^{\circ}$ & $5^{\circ}$ & $4^{\circ}$ & $5^{\circ}$ \\
\hline Geometría & Geometría & Operaciones & Operaciones \\
\hline Números & Números & Geometría & Geometría \\
\hline Operaciones & Operaciones & Números & Números \\
\hline
\end{tabular}

Las OM escogidas fueron:

\begin{tabular}{|l|l|l|}
\cline { 2 - 3 } \multicolumn{1}{c|}{} & \multicolumn{1}{c|}{$4^{\circ}$ Básico } & \multicolumn{1}{c|}{$5^{\circ}$ Básico } \\
\hline Números & Fracciones & Fracciones \\
\hline Operaciones & Problemas Multiplicativos & Problemas Multiplicativos \\
\hline Geometría & Cuadriláteros & Cuadriláteros \\
\hline
\end{tabular}

\section{En relación al primer ciclo básico}

La gestión de los procesos de enseñanza-aprendizaje observados en torno a la aritmética escolar resultó muy poco ambiciosa en términos de tareas matemáticas y objetivos de aprendizaje. Por lo general, no se fundamentaba totalmente en lo que proponen los actuales programas, ni tampoco en los libros de texto. Por ejemplo, en la mayoría de los procesos observados en torno al campo de problemas multiplicativos se redujo drásticamente la cantidad de tareas respecto a la propuesta curricular: de un total de 13 tareas identificadas en el programa, los profesores trabajaron sólo 4 ó 5 . Observamos que ninguno de los profesores hizo referencia a los procedimientos no convencionales para el cálculo de sumas y restas, ni tampoco para el cálculo de multiplicaciones y divisiones, sino que trabajaron directamente con el algoritmo convencional, pese a las reiteradas sugerencias dadas en los programas. En ninguno de los procesos observados relativos a este campo se estudió el tema de las comparaciones por cuociente y su relación con los problemas de comparación por diferencia, lo que habría permitido vincular las distintas operaciones. Sólo en un curso el docente dejó una tarea al respecto, pero que no fue retomada en la clase siguiente. Se observó un reducido espacio para el estudio y resolución de problemas aditivos y multiplicativos, los que aparecen sólo una vez que han sido estudiados los algoritmos convencionales correspondientes. Al centrar el estudio en el uso de los algoritmos aparece una marcada desarticulación entre las operaciones. Nos llamó la atención el hecho de que en dos de los procesos observados en $4^{\circ}$ básico los profesores usaban la caja Mackinder para trabajar problemas multiplicativos, pero la mayoría de sus alumnos recurrían inmediatamente al algoritmo tradicional, porque ya lo conocían. En 
estos casos los profesores solían recurrir a dibujos para explicar los problemas, lo que se transformaba más bien en un obstáculo para los estudiantes, puesto que la mayoría de ellos ya los había resuelto. Se trataba de una estrategia poco pertinente para el momento que vivía la clase, y que generó la pérdida de su objetivo.

En cuanto a los procesos observados en torno a las fracciones en $4^{\circ}$ básico, se detectó que, por lo general, los alumnos no presentaban demasiados inconvenientes en comprender el concepto de fracción como parte de un todo, que era el único modelo estudiado y, por ello, se gestionaba con bastante detenimiento y recursos que apoyaran su visualización a través de material concreto. Este modelo, aunque inicialmente útil para el aprendizaje, más tarde se convierte en un obstáculo epistemológico para el estudio de la fracción como medida, modelo que responde al problema originario que dio como resultado la construcción de los números fraccionarios. Para los niños de $1^{\circ}$ y $2^{\circ}$ ciclo básico, las fracciones existen en la medida en que el entero ha sido fraccionado en partes iguales, y no como cuantificadores con una medida más pequeña que la unidad.

De esta forma, detectamos que las OM's reconstruidas en los procesos de estudio observados en torno a la aritmética reproducen los aspectos esenciales del fenómeno de atomización de la actividad matemática descrito en investigaciones en didáctica anteriores (Espinoza et al., 2003). Los números naturales aparecen disociados de las fracciones, y las operaciones desarticuladas entre sí, a pesar de las claras directrices dadas en los programas para gestionar la articulación entre ellas.

\section{En relación al segundo ciclo básico}

Se detectó que, pese a la gran diversidad de estrategias de enseñanza observadas entre los distintos procesos de estudio analizados, es posible extraer una macrotécnica didáctica generalizada. En efecto, los profesores de $2^{\circ}$ ciclo privilegian el estudio de procedimientos y algoritmos para realizar ejercicios, por sobre el estudio de problemas y cuestiones que permitan comprender el sentido y significado de los conocimientos en construcción. Las explicaciones dadas por el profesor son las justas para que a los estudiantes les quede lo más claro posible cómo manipular las técnicas de cálculo. Hay casi una total ausencia de elementos teóricos que justifiquen el funcionamiento de las técnicas y que definan con precisión los objetos matemáticos que utilizan. En general la estrategia de enseñanza consiste en presentar a los alumnos los conocimientos matemáticos, especialmente las técnicas, mostrando con ejemplos cómo se utilizan frente a tipos de problemas bien determinados, para luego dar una lista de ejercicios de práctica referidos a contextos muy cerrados y alejados de las experiencias cotidianas de los niños. En esta estrategia, el papel de los estudiantes es permanecer en silencio, atentos para tomar apuntes de lo que hace el profesor y comprender sus explicaciones; en escasas oportunidades pueden hacer opciones intelectuales frente a las actividades que el profesor les presenta. Por lo general, dada la ausencia de tecnología y teoría en clases, las matemáticas son estudiadas en este ciclo como un conjunto de objetos aislados y desarticulados entre sí; los alumnos tienen serias dificultades para determinar qué conocimiento matemático es el que permite resolver un determinado problema, cuando éste es presentado dentro de una gama de problemas de distinto tipo. De esta forma, los alumnos a lo sumo llegan a aprender bien el manejo de las técnicas y a realizar cálculos, pero no aprenden a discernir frente a qué tipo de problemas son pertinentes. Por ello, tal como hemos señalado más 
arriba, los alumnos de $2^{\circ}$ ciclo van perdiendo paulatinamente la oportunidad de aprender a razonar matemáticamente.

Dados estos hallazgos en la sala de clases, encontramos varias contradicciones entre lo que los profesores declararon en sus entrevistas en relación a la matemática y su enseñanza y lo que efectivamente gestionaron en sus clases. Por lo general, en las entrevistas aludieron a la necesidad de dar más énfasis a las habilidades y competencias de los alumnos que a los contenidos propiamente tales, y de asegurar la significación o relevancia de los distintos temas matemáticos del currículum en conexión con la vida de sus estudiantes. En diversas ocasiones la mayoría de los profesores se refirió a la importancia de fomentar en los estudiantes habilidades tales como la capacidad para abstraer, experimentar, aprender a aprender, comunicar su trabajo y sus resultados, trabajar colaborativamente, resolver problemas, etc.

Como una forma de contrastar empíricamente nuestra hipótesis relativa al estancamiento del desarrollo de la actividad matemática de los niños a lo largo de la escuela básica, aplicamos un problema poco habitual para la cultura escolar de nuestro país pero que, con las herramientas con que cuentan los niños desde $3^{\circ}$ básico, es factible que puedan abordarlo y resolverlo. El problema se presenta tal cual muestra la figura 1. Los resultados que obtuvimos son sorprendentes, a la vez que desconcertantes, puesto que son mejores en los niños con menos escolaridad. A raíz de estos resultados quisimos indagar cómo estudiantes de enseñanza media, profesionales de diversos ámbitos (científico, tecnológico, humanista y comercial) y adultos escolarizados en general, abordaban el estudio de este problema.

En un trabajo publicado recientemente hemos presentado los principales hallazgos de esta experiencia (Espinoza et al., 2009).

Figura 1

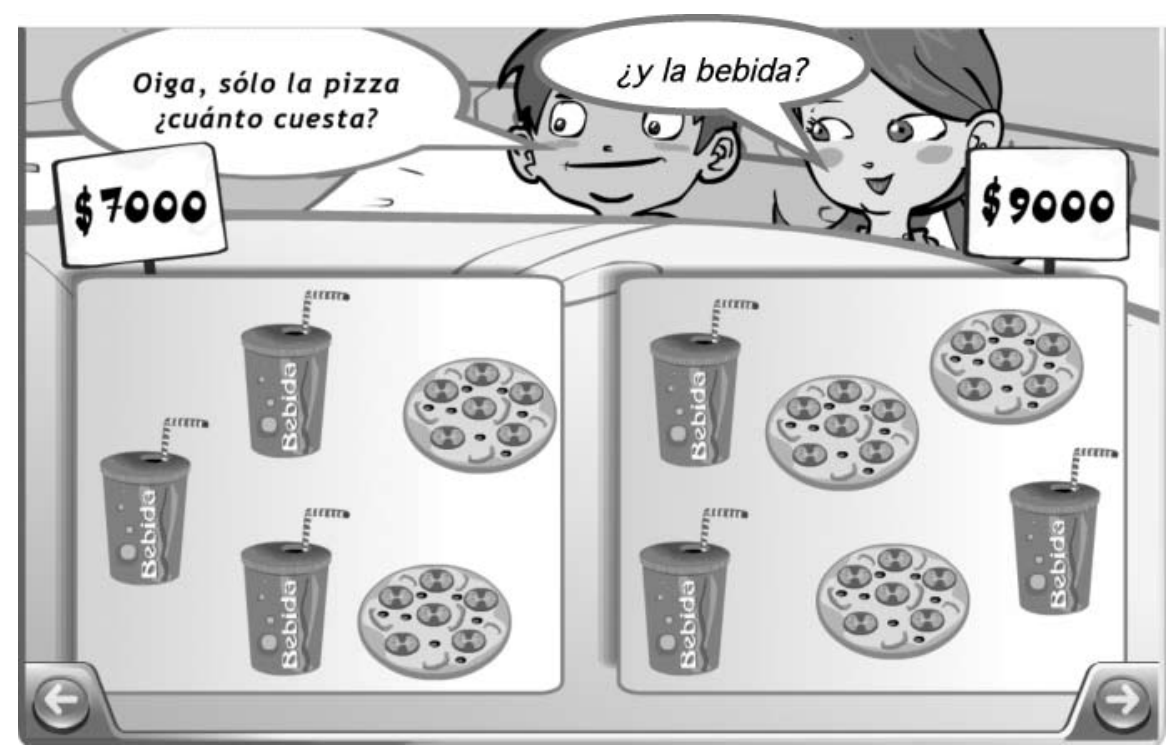




\subsection{ANALISIS DE CONDICIONES Y RESTRICCIONES INSTITUCIONALES QUE LAS ESCUELAS IMPONEN PARA EL DESARROLLO DE LOS PROCESOS DE ENSEÑANZA MATEMATICA EN AULA}

Dado que nuestra investigación se propuso estudiar el problema del escaso desarrollo de la actividad matemática que logran los estudiantes de enseñanza básica, en esta parte del estudio indagamos si existen factores de naturaleza institucional que podrían contribuir a explicar este fenómeno. Indagamos hasta qué punto la formas de organización de la enseñanza en ambos ciclos básicos son las mismas, y si existen diferencias entre las percepciones de los docentes de ambos ciclos, y de los directivos de las escuelas, sobre la matemática y su enseñanza. Para esto, en cada escuela se entrevistó a los directivos, y se realizó un focus group con todos los profesores de básica. A partir de los contratos didácticos determinados en el estudio de la dimensión fáctica para cada ciclo, y de toda la información recogida y analizada, fueron detectadas normas o patrones que parecían corresponder a restricciones institucionales. Presentamos a continuación una síntesis de los principales resultados obtenidos en esta tercera fase.

\section{Principales similitudes entre primer y segundo ciclo básico:}

- En toda la escuela básica, esto es, tanto en el $1^{\mathrm{er}}$ ciclo como en el $2^{\circ}$, pudo observarse que el grado de apropiación del currículo reformado es aún insuficiente. Los profesores han incorporado algunos planteamientos de la reforma a su discurso, pero los planteamientos básicos sobre cómo enseñar la matemática no los han incorporado a su práctica habitual y, en ciertos casos, ni siquiera los conocen. Así por ejemplo, los profesores señalaron en las entrevistas que es muy importante que los niños exploren; sin embargo, este momento del estudio rara vez fue observado en clases; también señalaron que la resolución de problemas es muy importante para la formación matemática de los alumnos, pero en escasas oportunidades se observó en las salas de clases, o se realizaba de manera inadecuada, considerando como problemas a unos ejercicios rutinarios que no requerían que los niños hicieran opciones intelectuales, sino la aplicación directa del conocimiento matemático que había sido recientemente enseñado por el profesor.

- En ambos ciclos se observó que la organización institucional de las prácticas de enseñanza por lo general no asigna suficiente importancia a la especialización de los profesores para asignarles sus cursos. Por lo general, los criterios predominantes para realizar dicha asignación son más bien de carácter administrativo y financiero.

- Los profesores de ambos ciclos tienen la oportunidad de ser bastante autónomos en cuanto a la planificación, ejecución y evaluación de sus clases, puesto que generalmente no existen desde la institución, ni directrices ni mecanismos efectivos de supervisión al trabajo docente. Esto, sumado a que los profesores no cuentan con herramientas suficientes para organizar y gestionar su trabajo de forma eficaz, redunda en una práctica muy variada que por lo general no cumple los estándares de calidad señalados en el currículum. La autonomía provista por la institución, en vez de favorecer el desarrollo profesional en forma personal y colectiva, lo dificulta, ya que dicha autonomía carece de las condiciones necesarias para ser aprovechada en beneficio de una actividad docente que se adecue a la especificidad de cada grupo de estudiantes para obtener mejores aprendizajes. 
- Todos los profesores observados, tanto en $1^{\text {er }}$ ciclo como en $2^{\circ}$, manifestaron tener carencias matemáticas importantes en relación a las exigencias curriculares del ciclo en que ejercen. Los instrumentos y medios existentes para apoyar la labor docente en el sistema, tales como libros de texto, material curricular de referencia y cursos de capacitación, suelen no ser suficientes para cubrir sus necesidades, ya sea porque no están a su alcance o porque no cumplen con el estándar de calidad necesario. En cuanto a los cursos de perfeccionamiento actuales, los profesores de ambos ciclos no los consideran adecuados, puesto que suelen existir dos tipos de tendencias en dichos cursos. Por un lado, están los que sobredimensionan la componente matemática por sobre la pedagógica y, por otro, los que priorizan la componente pedagógica en desmedro de la matemática. Esta polarización pone de manifiesto la compleja tarea de formar a profesores para que enseñen matemáticas de calidad.

- Los profesores de cada uno de los ciclos no cuestionan la metodología de enseñanza del otro. Por lo general, tanto los profesores de $1^{\text {er }}$ ciclo como los de $2^{\circ}$ consideran que sus formas de enseñar las matemáticas son adecuadas para las edades de los niños que tienen a su cargo, y diferentes de las que deben utilizar los profesores del otro ciclo. Por esta razón, ni unos ni otros manifiestan la necesidad de comunicarse entre ellos, salvo para resolver dudas matemáticas específicas.

- Tanto los profesores de $1^{\text {er }}$ ciclo como los de $2^{\circ}$ no saben cómo gestionar auténticos procesos de estudio matemático. No saben qué problemas plantear, de forma que provoquen en los alumnos la necesidad real de ampliar las herramientas matemáticas que conocen y que, a su vez, permitan que aparezcan diversas técnicas y, a partir de ellas, los elementos tecnológicos y teóricos correspondientes. De hecho, aunque la mayoría de los profesores de ambos ciclos manifestaron la importancia de incorporar la resolución de problemas a la enseñanza, en clases se limitaron al estudio de técnicas y algoritmos y a su aplicación en el desarrollo de ejercicios. De esta forma, los problemas no aparecen como el motor del proceso de estudio matemático, sino como una instancia para practicar y aplicar las técnicas previamente enseñadas por el profesor en contextos muy cerrados y poco reales, perdiendo de este modo su naturaleza.

\section{Principales diferencias entre primer y segundo ciclo básico:}

- Los profesores de $2^{\circ}$ ciclo tienen mayor relación con las matemáticas, y por ello, quizás menos temor a enseñarlas que los profesores de $1^{\mathrm{er}}$ ciclo, quienes por lo general se sienten poco competentes matemáticamente. Los profesores de $2^{\circ}$ ciclo suelen sentirse, a la vez que así son considerados por la comunidad escolar, como "los matemáticos de la escuela". Se suele recurrir a ellos ante cualquier duda relacionada con las matemáticas, como el cálculo de porcentajes, la construcción de escalas de notas, etc. Sin embargo, esta aparente superioridad no es suficiente para las demandas curriculares del ciclo. De hecho, los profesores de $2^{\circ}$ ciclo no tienen una formación matemática que les permita atribuirse esa consideración especial; la diferencia con sus pares de $1^{\text {er }}$ ciclo es que conocen un repertorio más amplio de técnicas y algoritmos, pero al igual que ellos suelen desconocer los fundamentos teóricos y tecnológicos que están detrás del funcionamiento de los mismos. Aparece aquí, más que una diferencia, una semejanza entre ambos ciclos. En efecto, los 
profesores de $2^{\circ}$ ciclo, al carecer de la tecnología y teoría matemática que justifica y hace comprensible el funcionamiento de las técnicas o procedimientos, no pueden establecer relaciones entre dichas técnicas, ni tampoco sus dominios de validez; de esta forma, la enseñanza de las matemáticas en este ciclo, al igual que en el $1^{\mathrm{er}}$, termina construyendo un conjunto de objetos matemáticos aislados entre sí, que nunca alcanzan a constituir una OM completa.

- Todos los profesores observados en $1^{\text {er }}$ ciclo manifestaron que deben ser protectores con los alumnos y respetar, por sobre todo, su condición de niños. Deben contestar siempre a sus preguntas, escribir todo en la pizarra, hacer trabajos grupales, actividades lúdicas que los entretengan, explicaciones concretas apoyadas en dibujos, objetos o imágenes cercanas a la realidad de ellos, etc. En $2^{\circ}$ ciclo, por el contrario, los profesores declaran que debe existir un cambio en la relación profesor-alumno, redefiniendo las cláusulas del contrato pedagógico en términos de una mayor participación del alumno en cuestiones de carácter formal, no relacionadas necesariamente con el estudio de las matemáticas. Los cambios más habituales son: se asigna trabajo individual en vez de grupal, se disminuye el uso de material concreto y de representaciones gráficas para apoyar la comprensión de los niños, y ya no se escribe todo en el pizarra, quedando en manos del alumno la tarea de registrar lo que el profesor dicta o explica.

- A los alumnos de $2^{\circ}$ ciclo se les hacen mayores exigencias que a los alumnos de $1^{\mathrm{er}}$ ciclo, pero sólo en términos del manejo más preciso y rápido de las técnicas. Así, mientras que en el $1^{\text {er }}$ ciclo se acepta y se valora que los niños usen procedimientos rudimentarios, poco precisos y de escasa eficiencia, en el $2^{\circ}$ ciclo se les exige que, frente a un tipo específico de problemas, utilicen "el" procedimiento impuesto por el profesor, y de la forma en que éste lo "enseñó". Así, hay una mayor exigencia para los alumnos de $2^{\circ}$ ciclo, quienes deben estudiar una matemática más rica y compleja que la del $1^{\text {er }}$ ciclo; sin embargo, tienen profesores con la misma formación y similares carencias.

- Los alumnos de $2^{\circ}$ ciclo consideran que un profesor es mejor en la medida que enseña de manera directa y clara las técnicas que se deben utilizar frente a un determinado tipo de problemas, sin recurrir a explicaciones teóricas sobre su funcionamiento. En $1^{\text {er }}$ ciclo, por el contrario, aunque la enseñanza de las matemáticas igualmente está centrada en el uso rutinario de técnicas, los alumnos no le dan a éstas la misma valoración. De esta forma se produce en el $2^{\circ}$ ciclo el efecto opuesto a lo deseado por la sociedad y el sistema educativo, esto es, que los niños aprendan a razonar matemáticamente. En efecto, el profesor centra su enseñanza en que sus alumnos aprendan a "hacer cosas" que puedan ser medidas colectivamente, es decir, productos visibles, y no da importancia al cuestionamiento de los medios (tecnología y teoría) que posibilitan la existencia de dichos productos. Los alumnos de $2^{\circ}$ ciclo dejan de explorar problemas y buscar sus propios medios de solución, ya que el profesor se los proporciona. La escuela, muy a su pesar, termina inhibiendo las potencialidades matemáticas inherentes a los alumnos y su capacidad de razonar matemáticamente.

- Los profesores de $2^{\circ}$ ciclo tienen mayor conocimiento de los programas oficiales que los profesores de $1^{\text {er }}$ ciclo, aunque esto no significa necesariamente una mayor incorporación de sus orientaciones en sus prácticas habituales. Esto puede deberse a que, en la mayoría de los casos, los profesores de $2^{\circ}$ ciclo deben hacerse cargo de la 
enseñanza de unos conocimientos matemáticos que conocen muy superficialmente, y cuentan con escasas herramientas para enfrentarla. Por esto es que suelen sentir una mayor necesidad que los profesores de $1^{\text {er }}$ ciclo por recurrir a documentos curriculares que circulan en el sistema, hacer cursos de capacitación, etc.

- Tanto los programas de estudio como los libros de texto de $1^{\text {er }}$ ciclo tienen mejor desarrollo desde la perspectiva didáctica que los de $2^{\circ}$ ciclo. No obstante, en el caso de los programas de estudio de $1^{\text {er }}$ ciclo, la densidad de la escritura y el formato de presentación hacen más compleja su lectura e interpretación que la de los programas de $2^{\circ}$ ciclo.

- Los profesores de $1^{\text {er }}$ ciclo imparten todas las asignaturas del ciclo, mientras que en el $2^{\circ}$ ciclo los profesores imparten sólo una o dos asignaturas. Los profesores de $2^{\circ}$ ciclo, que en su mayoría son generalistas, se van "especializando" en la práctica. Esta importante diferencia incide sobre la relación de los profesores con sus alumnos y con la disciplina que enseñan.

\section{CONCLUSIONES Y REFLEXIONES DERIVADAS DEL ESTUDIO}

El trabajo de investigación realizado, que abordó las dimensiones curricular, fáctica e institucional, nos permite postular lo que podría ser considerado un fenómeno didáctico por su grado de generalidad y su vinculación directa con una estrategia específica de enseñanza de las matemáticas (Brousseau, 1983). El fenómeno consiste en que en muchos casos, la escuela inhibe, de forma no intencionada y muy a su pesar, las potencialidades matemáticas que tienen los niños.

Pero entonces habría que preguntar imperiosamente: ¿qué pasa en la escuela que, lejos de ser su intención y propósito, termina limitando matemáticamente a muchos de sus alumnos? En función de los resultados de nuestra investigación podemos postular algunos factores que podrían contribuir a dar respuesta a esta preocupante cuestión:

- Hemos detectado que en la mayoría de las clases observadas se gestiona una enseñanza de las matemáticas alejada de sus sentidos y significados originarios; se clasifica los problemas de forma rígida y estandarizada y, con ello, se limita -en muchos casos se impide- la posibilidad de que los estudiantes exploren auténticamente posibles caminos de abordaje y solución. Se trata de una enseñanza relativamente arbitraria, que presenta los conocimientos matemáticos a los estudiantes a propósito de razones formales y no como respuesta a una necesidad. En este sentido, la enseñanza de las matemáticas enfrenta hoy día una importante dificultad: está instalada en el sistema escolar, y en particular en la escuela, una concepción de las matemáticas como un conjunto de conocimientos encerrados en sí mismos; es como si ella existiera por sí misma y para sí misma. En particular, se vuelve casi imposible hacer aparecer las matemáticas como algo que nace de lo no-matemático, como algo que matematiza realidades prematemáticas. La geometría de los matemáticos deja entonces de ser la matematización de los hechos espaciales, mientras que la aritmética deja de ser la matematización de los hechos relativos a la cantidad.

- Frente a un cierto tipo de problemas, generalmente se impone el uso de un único procedimiento para resolverlos, sin una justificación que permita valorarlo y descartar 
otros procedimientos. Hay que señalar también que los problemas en la escuela suelen ser ejercicios rutinarios, para cuya resolución es suficiente la mera aplicación del conocimiento previamente enseñado.

- Asimismo, la utilización de los procedimientos es por lo general muy rígida; las tareas se deben resolver de la misma forma en que el profesor lo mostró o "enseñó".

- Se observa una enseñanza atomizada de temas que, por lo general, no son articulados; no se realiza una construcción progresiva de los conocimientos, que se apoye en lo ya construido para incorporar nuevos conocimientos, necesarios para resolver nuevos problemas. La enseñanza del concepto de sucesor muestra de manera ejemplar esta situación. Tanto estudiantes como profesores de $2^{\circ}$ ciclo, frente a la pregunta de cuál es el sucesor de 6,5 responden sin titubear: 6,6. Esta concepción errada encuentra sus raíces en la forma en que se estudió el concepto de sucesor dentro de los números naturales, y en el hecho de que, abruptamente, se inicia el estudio de las fracciones y decimales sin establecer claras diferencias entre estos conjuntos numéricos y los naturales.

- La enseñanza no incorpora suficientemente lo que los niños ya saben, ni tampoco les otorga un verdadero rol dentro del proceso de construcción matemática. Por lo general, el papel del estudiante consiste en escuchar al profesor mientras "expone" la materia, copiar en sus cuadernos lo que el profesor dicta o escribe en la pizarra y reproducir los gestos realizados por el profesor al aplicar los conocimientos en la resolución de ejercicios. Suele haber escaso espacio para preguntas y para la interacción entre los estudiantes a propósito de las cuestiones matemáticas que están siendo estudiadas, y nunca participan en el proceso de validación de la producción de soluciones a las situaciones problemáticas abordadas, ni de los conocimientos empleados.

Esta manera de proceder está ligada a una concepción dominante en la cultura escolar, generalmente implícita, sobre la enseñanza de las matemáticas y, ligada a ésta, una concepción sobre qué son las matemáticas, cómo se construyen y cómo se pueden aprender, ya esbozada en párrafos anteriores. Ahora bien, existen varios obstáculos que dificultan el cambio de dicha concepción y, asimismo, la transformación de las prácticas. Lejos de ser un problema exclusivo del profesor, la insuficiencia de la enseñanza de las matemáticas se origina a propósito de múltiples factores de diversa índole, curricular, fáctico e institucional, y que se presentan en distintos niveles: micro (la sala de clases y la escuela), meso (el entorno más cercano a la escuela: comuna, departamento provincial de educación, familias) y macro (Ministerio de Educación, Universidades, la sociedad en su conjunto). En particular, existen ciertos mecanismos que se han puesto en funcionamiento para difundir los principios de la reforma que no contribuyen a modificar la situación de la enseñanza de las matemáticas. Así por ejemplo, se ha puesto gran acento en la innovación, entendiéndola en muchos casos como un conjunto de principios que se presentan en ruptura respecto de ciertas prácticas tradicionales. Se considera hoy que "hay que enseñar a resolver problemas" en oposición a la "enseñanza centrada en técnicas o algoritmos", o "se debe favorecer el trabajo grupal" en oposición al "trabajo individual", etc. Esta visión polarizada del proceso de enseñanza desvirtúa la esencia misma del aprendizaje, que requiere tanto de la resolución de problemas como de técnicas para resolverlos, del trabajo grupal como también del individual, etc. Ello conduce 
a poner de moda ciertos contenidos o énfasis muchas veces de manera hipertrofiada, sin realizar previamente un análisis sobre la necesidad, pertinencia y beneficio de incorporarlos. Por ejemplo, se ha puesto de moda desde la educación parvularia hasta el fin de la escolaridad obligatoria la resolución de problemas como contenido de enseñanza en sí mismo. Es posible encontrar capítulos enteros en libros de texto escolares dedicados a la enseñanza de la resolución de problemas de forma general y separada de cualquier actividad matemática específica.

Para el sistema educativo, es necesario que sus docentes dispongan de instrumentos y recursos que les sirvan para planificar y gestionar sus clases. Pero el aporte de esos recursos dependerá de las posibilidades reales de que dispongan los profesores para formarse en criterios de selección fundamentados científicamente, y participar en espacios institucionales de reflexión y formación para estudiarlos (OCDE, 2004). De esta forma, la implementación efectiva de cualquier nuevo currículo y la tarea de elevar el nivel de logro de aprendizaje de los estudiantes, en particular de enseñanza básica, debiera considerar una etapa de difusión y trabajo intenso con los docentes y con todos aquellos agentes del sistema educativo que participan en la tarea de la enseñanza.

\section{REFERENCIAS BIBLIOGRAFICAS}

Bosch, M., Espinoza, L. y Gascón, J. (2003). El profesor como director de procesos de estudio. Análisis de praxeologías didácticas docentes espontáneas, Recherches en Didactique des Mathématiques, 23 (1): 79-136.

Brousseau, G. (1983). Les obstacles epistémologiques et les problèmes en didactique, Recherches en Didactique des Mathématiques, 4 (2): La Pensée Sauvage.

Brousseau, G. (1986). Fondements et méthodes de la didactique des mathématiques, Recherches en Didactique des Mathématiques, 7 (2): La Pensée Sauvage.

Brousseau, G. (1990). Le contrat didactique: Le milieu, Recherches en Didactique des Mathématiques, 9 (3): 308-336.

Brousseau, G. et al. (1994). Concours externe de Recrutement des Professeurs d'Ecole. Université de Bourdeaux: Editorial LADIST.

Cox, C. (editor) (2003). Políticas educacionales en el cambio de siglo. La reforma del sistema escolar en Chile, Santiago: Editorial Universitaria.

Chevallard, Y. (1985). La transposición didáctica. Del saber sabio al saber enseñado, Buenos Aires: Aique. (La traducción es de 1997).

Chevallard, Y., Bosch, M. y Gascón, J. (1997). Estudiar Matemáticas: El eslabón perdido entre la enseñanza y el aprendizaje, Barcelona: ICE-Horsori.

Chevallard, Y. (1999). L'analyse des practiques enseignantes en théorie anthropologique du didactique, Recherches en Didactiques des Mathématiques, 19 (2): 221-265.

Chevallard, Y. (2004). Vers une didactique de la codisciplinariété. Notes sur une nouvelle épistemologie scolaire, Intervención en el Séminaire de Didactique des Mathématiques et de l'informatique, Grenoble, Université Joseph Fourier, Francia.

Espinoza, L. y Barbé, J. (2004). La Matemática en la Educación Básica y Media: Un análisis de discontinuidades entre ambos niveles educativos, Revista Chilena de Educación Matemática, 1: 77-90.

Espinoza, L., Barbé, J., Bosch, M. y Gascón, J. (2005). Didactic Restrictions on the Teaching of Limits of Functions at Secondary School, Educational Studies in Mathematics, 59: 235-268.

Espinoza, L., Barbé, J. y Gálvez, G. (2009). Estudio de fenómenos didácticos vinculados a la enseñanza de la aritmética en la educación básica chilena, Enseñanza de las ciencias, 2009, 27 (2). 
Leutenegger, F. (2000). Construction d'une "clinique" pour le didactique: une étude des phenómenes temporels de l'enseignement, Recherches en Didactique des Mathématiques, 18 (1): 34-56.

Ministerio de Educación (2004-2005). Orientaciones Nivel de Educación Básica, Santiago de Chile.

Ministerio de Educación (2007). Resultados Nacionales SIMCE 2006, Santiago de Chile.

PISA-Mineduc (2004). Competencias para la vida. Resultados de los estudiantes chilenos en el Estudio PISA 2000, Santiago de Chile.

OCDE (2004). Revisión de políticas nacionales de educación, Chile. Organisation for Economic Co-operation and Development (OECD), Paris.

TIMSS-Mineduc (2004). Chile y el aprendizaje de las matemáticas y ciencias según TIMSS, Santiago de Chile.

Sensevy, G. (1999). Eléments pour une anthropologie de l'action didactique. Note de synthèse pour l'habilitation à diriger des recherches. 
